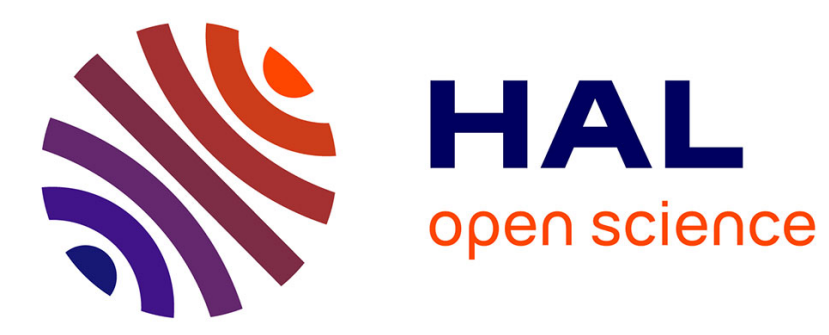

\title{
Note sur Camille Notton, traducteur de la chronique de Suvanna Khamdaeng
}

François Lagirarde

\section{To cite this version:}

François Lagirarde. Note sur Camille Notton, traducteur de la chronique de Suvanna Khamdaeng. Aséanie, Sciences humaines en Asie du Sud-Est, 2002, 9, pp.94-100. 10.3406/asean.2002.1075. halshs-02569735

\section{HAL Id: halshs-02569735 \\ https://shs.hal.science/halshs-02569735}

Submitted on 11 May 2020

HAL is a multi-disciplinary open access archive for the deposit and dissemination of scientific research documents, whether they are published or not. The documents may come from teaching and research institutions in France or abroad, or from public or private research centers.
L'archive ouverte pluridisciplinaire HAL, est destinée au dépôt et à la diffusion de documents scientifiques de niveau recherche, publiés ou non, émanant des établissements d'enseignement et de recherche français ou étrangers, des laboratoires publics ou privés. 
Note sur Camille Notton, traducteur de la chronique de Suvanna Khamdaeng

François Lagirarde

Citer ce document / Cite this document :

Lagirarde François. Note sur Camille Notton, traducteur de la chronique de Suvanna Khamdaeng. In: Aséanie 9, 2002. pp. 94100;

doi : https://doi.org/10.3406/asean.2002.1075

https://www.persee.fr/doc/asean_0859-9009_2002_num_9_1_1075

Fichier pdf généré le 07/09/2018 


\title{
Note sur Camille Notton, traducteur de la chronique de Suvanna Khamdaeng
}

\begin{abstract}
Camille Notton est l'exemple parfait de la victime plus que consentante du coup de foudre siamois, un phénomène apparemment décisif et infiniment reproductible. Né en 1881 à Peyrat de Bellac, dans le Limousin, c'est un digne fils d'instituteur de la III ${ }^{\mathrm{e}}$ République, capable et studieux. Après avoir fait son Droit à Paris et mené à bien l'apprentissage du chinois et du siamois, il est nommé en 1906 à l'ambassade de France à Bangkok comme élève interprète. Il s'y marie et devient consul de France à Chiang Mai pendant la première guerre mondiale, poste qu'il occupera pleinement jusqu'à la seconde. Il ne quitte la Thaïlande qu'en 1959 après avoir systématiquement refusé toute autre affectation en dehors de la capitale du Lanna.
\end{abstract}

Camille Notton sut s'entourer d'informateurs et de collaborateurs efficaces. L'un d'entre eux est resté dans la mémoire locale: M. Waghetzky, Russe blanc installé à Chiang Mai qui fut - paraît-il directement impliqué dans les travaux de recherche et de traduction de Notton ${ }^{18}$.

La personnalité de Camille Notton semble avoir été des plus remarquables et un chapitre lui est presque entièrement consacré dans un ouvrage bien connu dans le milieu des Affaires étrangères français ( $A u$ bout du Quai de Jacques Baeyens, publié à Paris en 1975 chez Fayard). Ces "souvenirs irrespectueux d'un diplomate" évoquent en particulier le style facétieux du consul de Chiang Mai ainsi qu'une mystification qui le rendirent célèbre.

On retiendra le fait que, dans son travail de traduction, Camille Notton se fait le défenseur de la langue et de la civilisation du Nord de la Thaïlande dont il constate l'affaiblissement sous les coups de boutoir du processus national de centralisation. Il demeure un pionnier incontournable des études sur le Lanna: ses travaux de traduction certes un peu datés ou perfectibles par endroits - continuent à faire référence dans la francophonie et au-delà. 


\section{Notes}

1. "TSK" ci-après pour évoquer ce texte sous ses deux versions disponibles. TSK est également connu sous le nom de chronique de la colonne Inthakhin (tāmnān sau indakhīla). Lorsque dans ces notes "chronique" apparaît en italiques il s'agit alors de la traduction de C. Notton. Nous remercions ici Louis Gabaude, Volker Grabowsky, Harald Hundius et Hans Penth d'avoir aimablement répondu à toutes nos questions à propos de cette chronique.

2. Ces ancêtres des khon müang ou Thaïs du Nord de la Thaïlande sont désignés par Thaïs (daiy) dans la chronique mais celle-ci étant de rédaction tardive on ne sait par quel ethnonyme ils se désignaient eux-mêmes au moment des événements décrits.

3. Le pilier se trouvait auparavant au Wat Sadü Müang ou Wat Inthakhin, aujourd'hui disparu, et fut déplacé au Wat Chedi Luang par le roi Kavila, si bien qu'il est impossible de connaître son aspect originel. Aujourd'hui, le pilier est constitué par un socle octogonal surmonté par une statue du Bouddha debout en position de réflexion, les mains légèrement croisées sur la poitrine. Le bâtiment n'est ouvert qu'une fois par an pour le festival du pilier qui a lieu au mois de mai ou juin.

4. La situation est peut-être moins désespérée qu'elle n'en a l'air puisque le professeur Harald Hundius m'assure avoir réalisé une copie de TSK en 1972 ou 1973 à partir d'un manuscrit regroupant différents textes dans une bibliothèque d'un monastère de Lamphun.

5. Deux autres chroniques, évoquent également l'origine de l'installation de la colonne d'Indra. Il s'agit de Tamnan chiang mai pang doem (Udom 2542b, 7201) et de Tamnan mahathera fa bot, traduite également par Notton sous le titre de Chronique du Mahāthera Fa Bot (Tanabe 2000, 314 note 9 pour plus de détails bibliographiques).

6. Hans Penth (1994a, 10-11) a fait justement remarquer qu'à cette époque de "renaissance" du Lanna la place des Lawa fut expressément reconnue: "when, in 1797 [...] the royal court moved back to Chiang Mai, Lawa were at the head of the ceremonial procession." Mais dans quel but?

7. Au moment de mettre sous presse ces "Notes" nous venons de découvrir un article du même Kraisri Nimmanhaeminda dans lequel cet incident est mentionné ("The irrigation laws of King Mengrai", p. 1, in Ethnographic notes on Northern Thailand, Lucien M. Hanks et al., Ithaca, New York, Cornell University, 1965).

8. Il n'est pas question ici du découpage selon les intertitres imaginés par C. Notton dans sa traduction.

9. Wiang Chet Lin fut-elle en réalité fondée bien après Chiang Mai elle-même (Sarasawadee 2543, 253, donne la date de 1411 E.C.) ou n'était-ce qu'une appropriation officielle d'un site lawa ou môn?

10. Cunda est un nom indien qui apparaît à plusieurs reprises dans la littérature pāli; il est intéressant de noter que deux Cunda y sont associés au cochon: un premier Cunda fut celui qui servit le curry de porc qui fut fatal au Bouddha tandis que l'autre est un Cunda boucher-charcutier (Cunda Sūkarika). Il est évoqué dans le commentaire du Dhammapada: au moment de sa mort, il aperçoit les flammes de l'enfer et il agonisera pendant sept jours, grognant comme les pourceaux qu'il avait l'habitude d'occire.

11. C. Notton a lu Nabbapurī, Sa-nguan Nabapurī, c'est-à-dire Navapurī, ville des "neuf". Nopburi en thaï.

12. Pourrait-il s'agir du pays môn ou de Sukhothai? 
13. On ne peut que renvoyer ici aux pages de G. Condominas intitulées "Notes sur l'histoire lawa", d'abord publiées en 1974 dans Art and Archeology in Thailand par le Département des Beaux-Arts à Bangkok puis dans L'espace social à propos de l'Asie du Sud-Est.

14. Dans l'histoire de Sukhotai, le nom a été porté par quatre rois entre 1346 et 1438.

15. Ses statues sont nombreuses. La plus célèbre se trouve bien entendu au Wat Phra Boromathat Doi Suthep mais aussi au Wat Phra That Doi Kham, etc. (Kraisri Nimmanhaeminda 1967, 188).

16. Notton l'appelle Ba Muttalang et Sa-nguan Mamuttalang (māmuttaḥlāñ).

17. Données statistiques recueillies par P. Pichard pour le projet "Monastère bouddhique" (EFEO, Bangkok).

18. On trouvera dans le Bulletin de l'Alliance française de Bangkok une série d'articles biographiques consacrés à Camille Notton ainsi qu'une bibliographie de ses œuvres (Maurice Bouchet, $B A F B$, mai, juin, juillet, août 1988). La maison de Notton, ancienne légation de France à Chiang Mai, abrite aujourd'hui l'Alliance française de Chiang Mai.

\section{Références bibliographiques}

\section{Anan GANJANAPAN}

2542 (1999) - "Phithi wai phi muäng lae amnat rat nai lanna" (Wai Pii Muang and the State's Power in Lanna), Sangkhom lae vatanatham nai prathet thai (Thailand: Culture and Society), Bangkok, Princess Maha Chakri Sirindhorn Anthropology Centre, p. 150-161.

\section{Aroonrut WICHIENKEEO}

2000 - "Lawa (Lua): A Study from Palm-Leaf Manuscripts and Stone Inscriptions" p. 122-138 in Dynamics of Ethnic Cultures Across National Boundaries in Southwestern China and Mainland Southeast Asia: Relations, Societies and Languages, (HAYASHI Yukio et YANG Guangyuan éd.) Chiang Mai, Ming Muang Printing House.

\section{CONDOMINAS, Georges}

1980 - L'espace social à propos de l'Asie du Sud-Est, Paris, Flammarion.

\section{DifFLOTH, Gérard}

1984 - The Dvaravati Old Mon Language and Nyah Kur, Bangkok, Chulalongkorn University Printing House.

Kraisri NIMMAHAEMINDA

1967 - "The Lawa guardian spirit of Chiengmai"

Journal of the Siam Society, volume LV part 2, p. 185-225. 
LeBAR, Frank M.; HickeY, Gerald C. et Musgrave, John K. 1964 - Ethnic groups of mainland Southeast Asia, New Haven, Human Relations Area Files Press.

NotTon, Camille 1926 - Annales du Siam, première partie. Chroniques de: Suvanna Khămdëng, Suvanna K'ôm Khăm, Sinhanavati, Paris, Limoges, Nancy, Imprimerie Charles Lavauzelle \& $C^{\text {ie }}$.

Mani PHAYOMYONG 2542 (1999) - "Inthakhin" [Inthakhin (Indakhila)], Saranukrom Watanathamthai phak nüa [Encyclopédie de la culture thaïe, région Nord], volume 15, p. 7927-7928.

PENTH, Hans

1994a - A brief history of Lān Nā: civilizations of North Thailand, Chiang Mai, Silkworm Books.

1994b - Jinakālamālì Index, Oxford et Chiang Mai, the Pali Text Society et Silkworm Books.

RAENDCHEN, Oliver 2000 - "The Tai lak: ritual and socio-political function" Proceedings of the International Conference on Tai Studies July 29-31, 1998, Bangkok, Institute of Language and Culture for Rural Development, Mahidol University p. 223-240.

Sa-nguan (Sanguan) CHOTISUKHARAT

1971 - "Supernatural beliefs and practices in Chiengmai", Journal of the Siam Society, volume 59, part 1, 211-231.

2515 [1972] - Prachum tamnan lanna thai [Recueil des chroniques du Lanna thaï], Bangkok, Odeon Store (en thaï).

Sarasawadee ONGSAKUL

2529 [1986] - Pravatisat Lanna [Histoire du Lanna] (3e édition 2544 [2001]),Bangkok: Samnakphim Amarin (en thaï). 2543 [2000] - Chumchon boran nai aeng chiang mai lamphun (Ancient Communities in the Chiang Mai - Lamphun Basin), Bangkok, Amarin Book Center (en thaï avec un résumé en anglais).

Sommai PrEMchit et Amphay DorÉ

1992 - The Lan Na Twelve-Month Traditions, Chiang Mai, The Toyota Foundation.

SWEARER, Donald K.

1967 - "Myth, legend and history in the northern Thai Chronicles", Journal of the Siam Society volume 62 part 1, p. 65-88. 1995 - The Buddhist World of Southeast Asia, Albany, State University of New York Press. 
TANABE, Shigeharu

2000 - "Autochtony and the Inthakin Cult of Chiang Mai", Civility and Savagery: Social Identity in Tai States, Andrew Turton (ed.), Richmond, Surrey, Curzon Press, p. 294-318.

TERWIEL, B.J.

1978 - "The origin and meaning of the Thai 'City Pillar'", in Journal of the Siam Society volume 66 part 2, p. 159-171.

Udom RUNGRÜANGSI

2542a [1999] - "Suvanna khamdaeng, chao" [Suvanna Khamdaeng, prince], Saranukrom watanathamthai phak nüa [Encyclopédie de la culture thaïe, région Nord], volume 13, p. 7086-7087.

2542b [1999] - "Sao inthakhin" [le pilier Inthakhin], Saranukrom Watanathamthai phak nüa [Encyclopédie de la culture thaïe, région Nord], volume 14, p. 7201-7203.

WIJEYEWARDENE, Gehan

1986 - Place and Emotion in Northern Thai Ritual Behaviour, Bangkok, Pandora.

WyatT, David. K. et Aroonrut WiChIENKEEO

1995 - The Chiang Mai Chronicle, Chiang Mai, Silkworm Books.

YouNG, Gordon

1961 - The Hill Tribes of Northern Thailand, Bangkok, the Siam Society.

François Lagirarde

École française d'Extrême-Orient Bangkok 


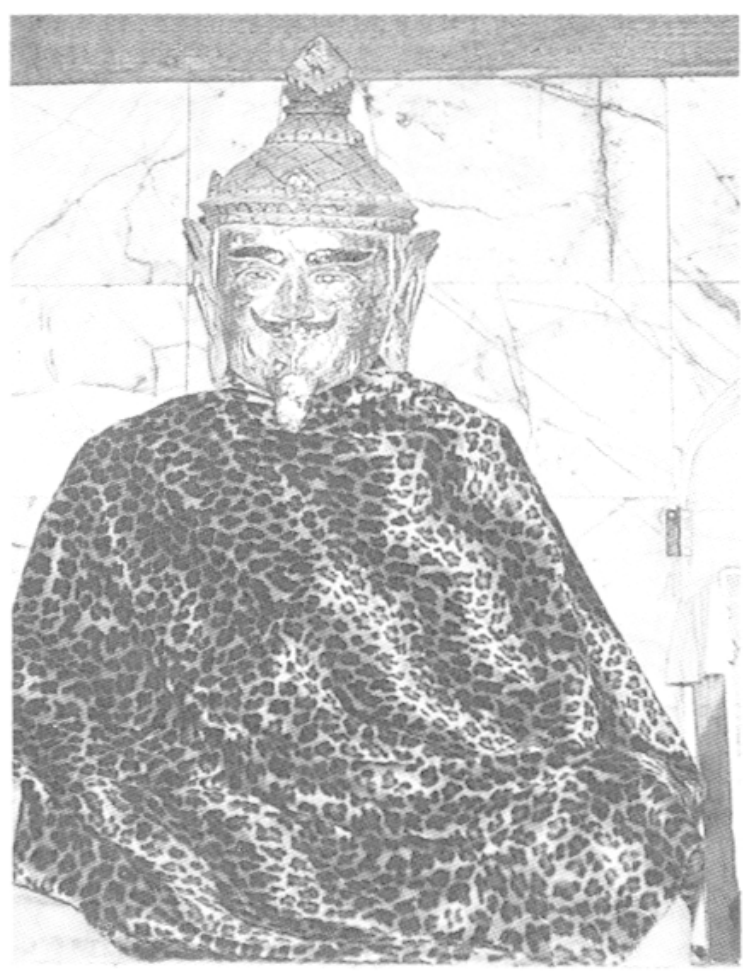

5 - L'ermite de Doi Suthep au Wat Phra Boromathat Doi Suthep

(ph. L. Gabaude)

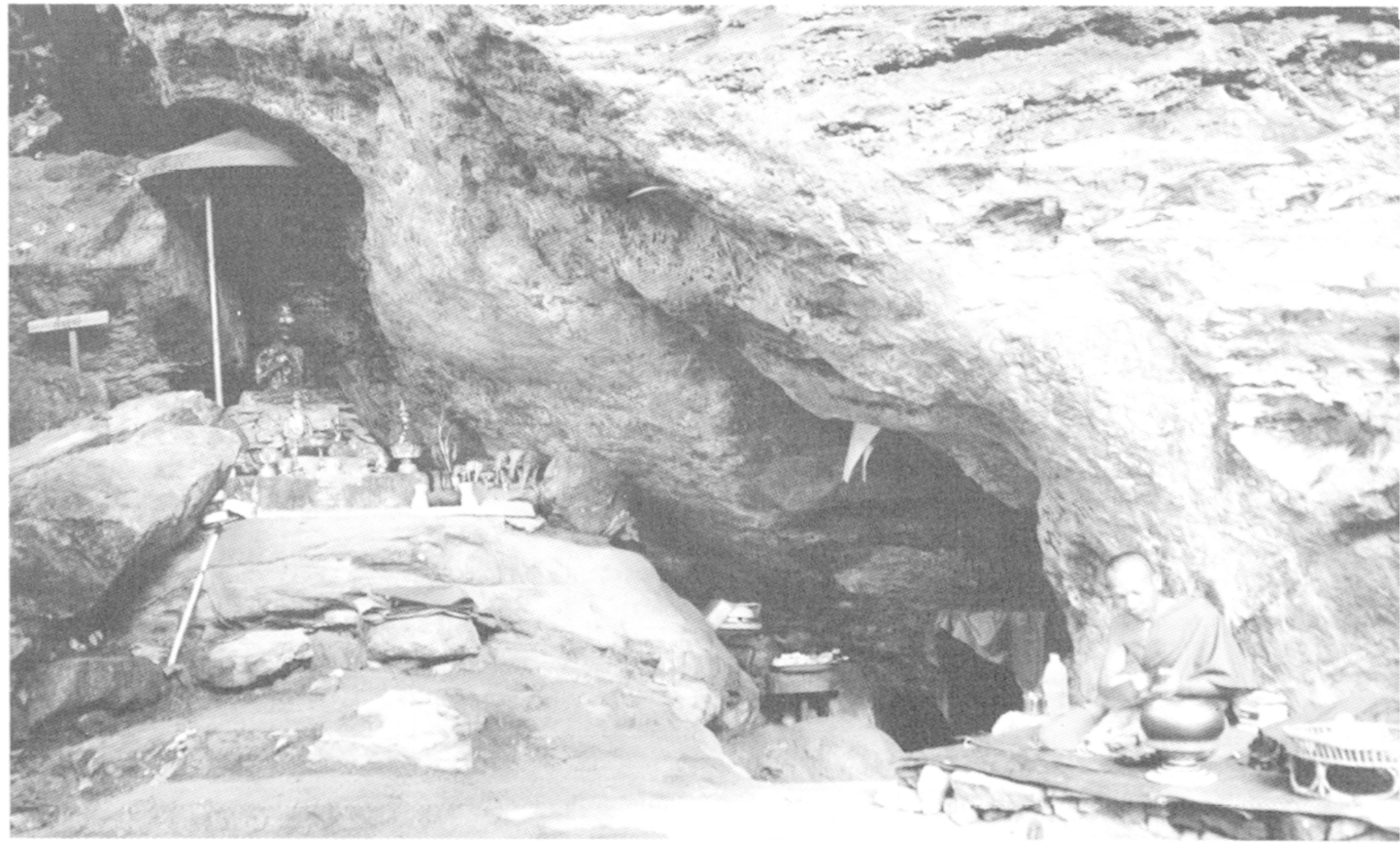

6 - Tham rüsi, la grotte de l'ermite près du Wat Phra Boromathat Doi Suthep

(ph. L. Gabaude) 
La Chronique de Suvanna Khămdëng est le premier texte historique traduit par Camille Notton dans ses Annales du Siam publiées entre 1926 et 1932, en trois parties. Dans le premier volume figurent également la Chronique du Mahathera Fa Bot, la Chronique de Suvanna Kh'ôm Khăm et la Chronique de Sinhanavati. Les deux volumes suivants contiennent la Chronique de La:p' un (vol. II, 1930, connue également sous le titre de Cāmadevivarisa) et la Chronique de Xieng Mai (vol. III, 1932).

Les textes des Annales du Siam (vol. I) sont précédés d'une préface, d'une table des abréviations, d'un tableau des systèmes de transcription, d'une chronologie, d'une liste des manuscrits et d'une table des illustrations. Nous mentionnons ci-dessous ce qui demeure pertinent à la Chronique de Suvanna Khămdëng.

Dans sa préface, $C$. Notton rappelle que les trois chroniques présentées dans ce volume sont des textes en thaï du Nord (yuan ou phasa phïn müang) qui s'intéressent "à la période la plus reculée de l'histoire des T'ài". C. Notton signale qu'il est d'ailleurs le premier à traduire ces chroniques dans leur intégralité car les éditions en siamois établies au début du $\mathrm{XX}^{\mathrm{e}}$ siècle n'offrent en effet que des traductions abrégées, sélectives. Ainsi, il souligne avec tact, en bon diplomate qu'il entend rester, le traitement incomplet ou partial que les savants de Bangkok ont fait subir à la littérature du Lanna. Il avoue qu'il n'a pas pu travailler sur des copies anciennes et qu'il n'a obtenu que des documents "encore récents" en 1925. Mais C. Notton ne donne pas d'indications précises sur ce qu'il entend par "récent".

Sa table des abréviations est la suivante:

Signe "-" avant un nombre d'années: Avant l'ère du Christ.

B. S. Buddhasakarāja, ère du Buddha.

C. S. Culasakarāja, petite ère.

Alabaster The Wheel of the Law, par Henry Alabaster.

BEFEO Le Bulletin de l'École Française d'Extrême-Orient.

BURNOUF Le Dictionnaire Classique Sanscrit-Français, par Emile BURNOUF.

ChILDERS A Dictionary of the Pali Language, par Robert Ceasar CHILDERS.

Gazetteer Gazetteer of Upper Burma and the Shan States, par J. George SCOTT.

GERINI, Chŭla Chŭlăkantamanigala or The Tonsure Ceremony, par G.-E. GERINI.

GERINI, Researches Researches on Ptolemy's Geography of

Eastern Asia, par Colonel G.-E. GERINI.

PALlEGoIX Dictionnaire Siamois-Français-Anglais, par D.-J.-B. PALlegoix, revu par J.-L. VEY.

P'ong. Yônok Rüang P'ongsawadan Yônok, par P'ra:ya Prajākicakaracakra.

PP P'ra:ya Prajākicakaracakra.

Wells WILliams A Syllabic Dictionary of the Chinese Language, par S.WELLS WILliams.

Les systèmes de transcription utilisés pour le pāli, le thaï du Nord (yuan) et le siamois sont présentés dans un tableau d'ailleurs incomplet pour les langues thaïes. Les consonnes basses aspirées (kho khwai, tho tahan, pho phan) sont notées à l'aide d'une apostrophe, sans " $h$ ". Pour le pāli le système utilisé est celui de R. C. Childers. Ce tableau est suivi d'indications chronologiques qui fournissent le nom des ères locales ("la Buddha Sakarāja qui commence en - 543" et "la Culasakarāja commence en 638 A.D.") et le nom des mois en thaï du Nord.

Aucune indication n'est donnée sur l'origine des manuscrits traduits.

Les illustrations originales relatives à la Chronique de Suvannı Khămdëng sont au nombre de trois. La première, intitulée “Types de race Lua:", représente un groupe de Lawa. La seconde est une vue du Wat Phan Takoeun à Chiang Mai (aujourd'hui Wat Chai Siphum) et la troisième une vue de l'édifice renfermant la colonne Indakhîla au Wat Chedi Luang à Chiang Mai.

Dans cette réimpression les notes ont été replacées en fin d'ouvrage en numérotation continue. Elles étaient en bas de page dans l'édition originale. Les intertitres sont de C. Notton. 